\title{
A Geometrical Presentation of the Surface Mapping Class Group and Surgery
}

\author{
S. Matveev ${ }^{1}$, M. Polyak ${ }^{2, \star}$ \\ 1 Department of Mathematics, Chelyasbinsk State University, Chelyabinsk 454136, Russia \\ 2 Department of Mathematics, University of California at Berkeley, Berkeley CA 94720 and MSRI, \\ 1000 Centennial Drive, Berkeley CA 94720, USA
}

Received: 1 February 1993

\begin{abstract}
We construct a tangle presentation of the mapping class group similar to a natural presentation of the braid group by geometrical braids. A relation between surgery and Heegard diagrams for 3-manifolds arising in this way and different applications are studied.
\end{abstract}

\section{Introduction}

It is well-known that the mapping class group of the disc with $n$ marked points has a natural presentation as the group of geometrical braids with $n$ strings. We give a similar presentation of the mapping class group of an orientable surface of arbitrary genus (which may also easily be generalized for the case of a surface with marked points). A relation between surgery presentation of 3-manifolds and Heegard diagrams (see $[6,11]$ ) arising in this way is investigated. This relation enables us to prove that if a 3-manifold has Heegard decomposition of genus two, it may be obtained by surgery on a framed arborescent link in $S^{3}$. We also provide a new proof (similar in spirit to [7]) of Kirby's theorem [5], which in our setting is an easy consequence of stable equivalence of Heegard splittings and Wajnryb's presentation for the mapping class group of a surface [13].

The paper is organized in the following way: in Sect. 1 we recall the notion of framed $2 n$-tangles and their diagrams. In Sect. 2 Kirby calculus for framed $2 n$-tangles is introduced. Section 3 is devoted to the definition of the group $T_{2 n}$ of admissible $2 n$-tangles. We state our main theorem in Sect. 4; the proof is given in Sects. 5, 6. In Sects. 7, 8 we study the relation between surgery and Heegard decompositions. As a corollary of our construction in a particular case of Heegard genus two we obtain (in Sect. 7) the result mentioned above. A new proof of Kirby's theorem is established in Sect. 9.

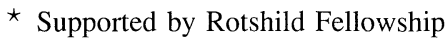


The paper was written when the first author was visiting Tel-Aviv University at the beginning of 1992. He would like to express his gratitude to Prof. M. Farber for his friendly support during the visit.

\section{Framed $2 n$-Tangles}

For a given integer $n \geq 0$ let $Y_{2 n}$ be a set of $n$ pairs $\left\{i^{-}, i^{+}\right\}, 1 \leq i \leq n$ of different points in the $x y$-plane $\mathbb{R}^{2} \subset \mathbb{R}^{2} \times \mathbb{R}^{1}$. To make our choice explicit we will put $i^{ \pm}=(0, i \pm 1 / 4)$. By a $2 n$-tangle we mean a proper one-dimensional submanifold of $\mathbb{R}^{2} \times[0,1]$ such that its boundary coincides with the set $Y_{2 n} \times\{0,1\}$. A framing of $2 n$-tangle is a trivialization of its normal bundle. We require that the restriction of the framing to $Y_{2 n} \times\{0,1\}$ should be induced by the standard $x y$-structure of $\mathbb{R}^{2}$ (say, in the positive direction of $y$-axis).

Given two framed $2 n$-tangles $\xi$ and $\zeta$ one may define their product $\xi \cdot \zeta$ to be $2 n$-tangle obtained by gluing the top of the "squeezed" copy $\zeta^{\prime} \subset \mathbb{R}^{2} \times[0,1 / 2]$ of $\zeta$ to the bottom of the squeezed copy $\xi^{\prime} \subset \mathbb{R}^{2} \times[1 / 2,1]$ of $\xi$.

Each $2 n$-tangle can be presented by tangle diagram, i.e. by its (general position) projection to $\mathbb{R}^{1} \times[0,1]$ with over- and underpasses in each crossing point indicated in the usual way. Tangle diagram determines the framing induced by the vector field orthogonal to $\mathbb{R}^{1} \times[0,1] \subset \mathbb{R}^{2} \times[0,1]$. Two tangle diagrams represent the same framed $2 n$-tangle iff they are regularly isotopic. The addition of a kink changes the framing of corresponding component by \pm 1 . It will be convenient to replace positive and negative kinks by small white and black circles respectively. A pair of opposite kinks on the same string of the tangle may by cancelled, as illustrated in Fig. 1.

Fig. 1.
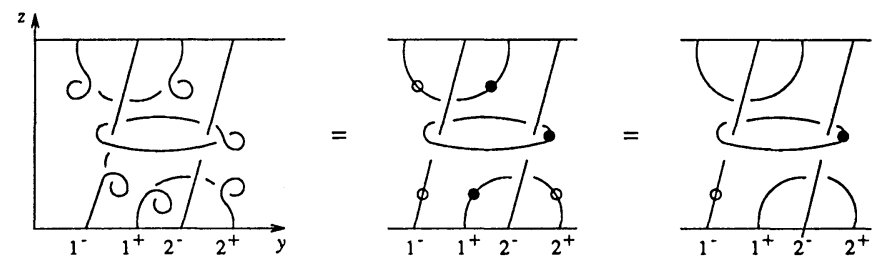

\section{Kirby Moves}

In [5] Kirby introduced two operations $O_{1}, O_{2}$ on framed links in a sphere $S^{3}$, later called Kirby moves. Denote by $\chi\left(M^{3}, L\right)$ the result of Dehn surgery of a 3-manifold $M^{3}$ along a framed link $L \subset M^{3}$. Then the following holds:

Kirby Theorem [5]. Given two framed links $L_{1}, L_{2} \in S^{3}$ one can pass from $L_{1}$ to $L_{2}$ by a sequence of moves $O_{1}, O_{2}$ iff $\chi\left(S^{3}, L_{1}\right)$ is homeomorphic to $\chi\left(S^{3}, L_{2}\right)$ (by an orientation preserving homeomorphism).

We extend the Kirby moves to the operations on a framed $2 n$-tangle $\xi \subset \mathbb{R}^{2} \times[0,1]$ by introducing the following moves $K_{1}-K_{3}$ :

$K_{1}$ : Add to $\xi$ an unknotted \pm 1 framed circle separated from the other strings of $\xi$ by an embedded 2 -sphere $S^{2} \subset \mathbb{R}^{2} \times[0,1]$. This move coincides with the Kirby move $O_{1}$. 


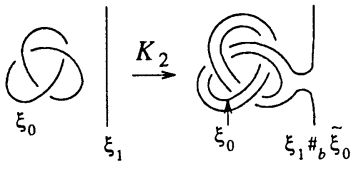

Fig. 2 .

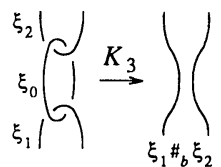

Fig. 3.

$K_{2}$ : Let $\xi_{0}$ be a closed component of $\xi$. Add $\xi_{0}$ to $\xi_{1}$ by replacing $\xi_{1}$ with $\xi_{1} \#_{b} \tilde{\xi}_{0}$, where $\#_{b}$ is a band connected sum and $\tilde{\xi}_{0}$ is obtained by pushing $\xi_{0}$ off itself along the framing, as illustrated in Fig. 2. If $\xi_{1}$ is closed, this move coincides with the Kirby move $\mathrm{O}_{2}$.

$K_{3}$ : Let $\xi_{0}$ be a closed, 0 -framed component of $\xi$ bounding an embedded disk $D \in \mathbb{R}^{2} \times[0,1]$ which intersects with $\xi \backslash \xi_{0}$ in exactly two points belonging to different components $\xi_{1}, \xi_{2}$ of $\xi$. Suppose also that either at least one of the components $\xi_{1}, \xi_{2}$ is closed or $\partial \xi_{1} \subset \mathbb{R}^{2} \times\{0\}, \partial \xi_{2} \subset \mathbb{R}^{2} \times\{1\}$. Then we may replace $\xi_{0} \cup \xi_{1} \cup \xi_{2}$ by $\xi_{1} \#_{b} \xi_{2}$, where the band $b$ intersects $D$ along the middle line of $b$, see Fig. 3 .

Definition. Two framed $2 n$-tangles are said to be $K$-equivalent, if one can pass from one to another by a (finite) sequence of moves $K_{1}^{ \pm 1}, K_{2}, K_{3}^{ \pm 1}$; we denote it by $\xi \underset{K}{\sim}$.

It is convenient for our further purposes to introduce some additional moves $K_{4}, K_{5}$ (which can be expressed via $K_{1}^{ \pm 1}, K_{2}, K_{3}^{ \pm 1}$ ).

The move $K_{4}$ is the deletion of unknotted \pm 1 -frame circle at the expense of the full left- or right-hand twist on the strings linked with it [4], as shown in Fig. 4.

Let $\xi_{0}$ be a closed, 0 -framed component of $\xi$ which bounds an embedded disk $D \subset \mathbb{R}^{2} \times[0,1]$. Suppose that $D \cap\left(\xi \backslash \xi_{0}\right)$ consists of exactly one point lying on some component $\xi_{1} \subset \xi$. Then the move $K_{5}$ is the deletion of components $\xi_{0}, \xi_{1}$ as illustrated in Fig. 5.

Some remarks should be made at this stage.

Remark 2.1. The move $K_{4}$ may be expressed via $K_{1}^{ \pm 1}$ and $K_{2}$, see [4]. The same is true for the move $K_{5}$. To obtain this, note that due to presence of $\xi_{0}$ we may change overcrossings of $\xi_{1}$ with itself and with other components of $\xi$ to undercrossings by means of $K_{2}$; this allows us to unlink (and unknot) $\xi_{1}$ so that we may consider $\xi_{0}$ and $\xi_{1}$ to form the Hopf link far from the other components of $\xi$. We may also put the framing of $\xi_{1}$ to be +1 by adding \pm 1 -framed unknotted circle (by $K_{1}$ ) and link it with $\xi_{1}$ (as above), which in view of $K_{4}$ is equivalent to changing the framing of

Fig. 4.

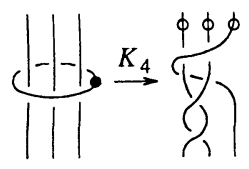

Fig. 5.

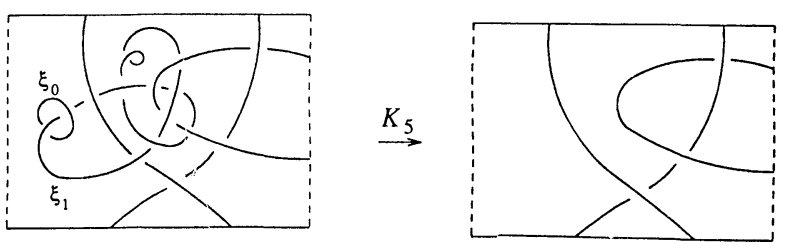


$\xi_{1}$ by \pm 1 . It remains to take note that now the deletion of $\xi_{0} \cap \xi_{1}$ can be carried out by means of $K_{4}$ and $K_{1}^{-1}$.

Remark 2.2. If at least one of the components $\xi_{1}, \xi_{2}$ (say $\xi_{1}$ ) in the definition of the move $K_{3}$ is closed, then $K_{3}$ may be expressed via $K_{1}^{ \pm 1}$ and $K_{2}$. Actually, it is a composition of the move $K_{2}$ (we add $\xi_{1}$ to $\xi_{2}$ along the band $b$ ) and the move $K_{5}$.

Remark 2.3. Any framed link $L \in S^{3}$ may be considered (after isotopy into $\mathbb{R}^{2} \times[0,1] \subset \mathbb{R}^{3} \in S^{3}$ ) as a framed $2 n$-tangle for $n=0$, hence the moves $K_{1}^{ \pm 1}, K_{2}, K_{3}^{ \pm 1}$ can also be applied to links. The equivalence relation $\underset{K}{\sim}$ for links coincides with the equivalence $\underset{\partial}{\sim}$ of Kirby [5].

\section{Admissible 2n-Tangles}

Unfortunately the multiplication of $2 n$-tangles does not agree with the equivalence relation $\underset{K}{\sim}$ : it may occur that $\xi \underset{K}{\sim} \zeta$, but $\xi \gamma$ is not $K$-equivalent to $\zeta \gamma$ for some $2 n$ tangle $\gamma$. The reason is that the condition on $\xi_{1}, \xi_{2}$ in the definition of the move $K_{3}$ may be violated after multiplication by $\gamma$. To avoid this difficulty we introduce the notion of an admissible $2 n$-tangle.

Note that each geometrical $2 n$-braid $\xi$ with the ends at $Y_{2 n} \times\{0,1\}$ can be considered as a $2 n$-tangle. The braid $\xi$ is called admissible, if the corresponding permutation preserves the decomposition of $Y_{2 n}$ into (unordered) pairs. More precisely we require that if for some $i, j$ a string of the braid runs from $i^{-} \times\{0\}$ to $j^{ \pm} \times\{1\}$, then the other string has to run from $i^{+} \times\{0\}$ to $j^{\mp} \times\{1\}$. An arbitrary framing of the braid is allowed.

Let $\beta_{\imath}^{ \pm 1}, 1 \leq i \leq n$ be the framed $2 n$-tangles depicted in Fig. 6 .

Fig. 6 .

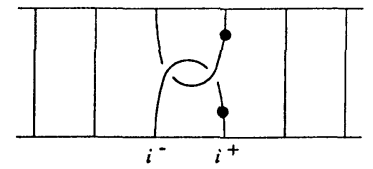

$\beta$

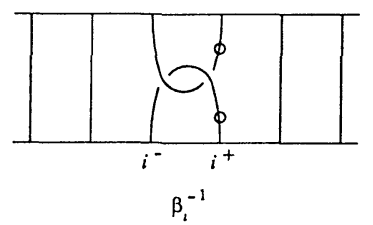

$\beta_{i}^{-1}$

Definition. A framed $2 n$-tangle $\xi$ is called admissible, if it can be written as $\xi=$ $\xi_{1} \xi_{2} \ldots \xi_{k}$ (for some $k$ ) where each $\xi_{i}$ is either $\beta_{\imath}^{ \pm 1}, 1 \leq i \leq n$ or a framed admissible $2 n$-braid.

Note that each admissible $2 n$-tangle $\xi$ satisfies the following

Condition $(*)$. For each unclosed component $\xi_{0}$ of $\xi$ one of the following holds:

(i) $\partial \xi_{0}=\left(i^{-} \cup i^{+}\right) \times\{0\}$ for some $i$, or

(ii) $\partial \xi_{0}=\left(i^{-} \cup i^{+}\right) \times\{1\}$ for some $i$, or

(iii) $\partial \xi_{0}=i^{ \pm} \times\{0\} \cup j^{ \pm} \times\{1\}$ for some $i, j$ and there exists another component $\xi_{1}$ of $\xi$ such that $\partial \xi_{1}=i^{\mp} \times\{0\} \cup j^{\mp} \times\{1\}$.

One may easily see that multiplication of tangles and the moves $K_{1}-K_{5}$ preserve the condition $(*)$. This implies that the multiplication of admissible $2 n$-tangles determines correctly defined multiplication on $K$-equivalence classes of admissible $2 n$-tangles. 
Denote by $T_{2 n}$ the semigroup of $K$-equivalence classes of admissible $2 n$-tangles. Proposition 3.1. $T_{2 n}$ is a group.

Proof. Immediately follows from the fact that the reflection of an admissible $2 n$ tangle $\xi$ with respect to the middle plane $\mathbb{R}^{2} \times\{1 / 2\}$ produces the inverse $2 n$-tangle $\xi^{-1}$.

\section{Main Theorem}

Let $\Sigma_{n, 1}$ be an orientable surface of genus $n$ with one boundary component. The mapping class group $M_{n, 1}$ of $\Sigma_{n, 1}$ is the group of isotopy classes of orientationpreserving self homeomorphisms of $\Sigma_{n, 1}$ fixing the boundary pointwise. We will oftenly identify $M_{n, 1}$ with the mapping class group of a surface $\partial H_{n}$ with an open disk $D \in \partial H_{n}$ removed, where $H_{n}$ is the standard solid handlebody of genus $n$ in $\mathbb{R}^{3} \in S^{3}$.

Denote by $a_{i}, b_{i}, d_{i}, e_{i}, 1 \leq i \leq n$ the right-hand Dehn twists with respect to curves labeled, by abuse of notation, with the same letters, as shown in Fig. 7 (notice that $a_{1}=d_{1}=e_{1}$ ). Denote by $\alpha_{i}, \beta_{i}, \varepsilon_{\imath}$ and $\delta_{i}, 1 \leq i \leq n$ the admissible $2 n$-tangles depicted in Fig. 8, 6 (put $\alpha_{1}=\delta_{1}$ ). Clearly, $\varepsilon_{i}$ is admissible in view of $K_{4}$ and $\delta_{1} \underset{K}{\sim} \varepsilon_{1}$.

Fig. 7.

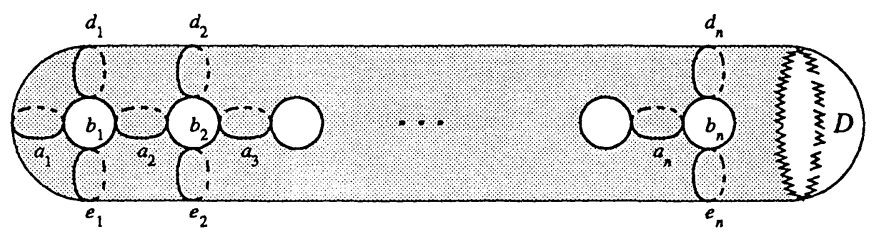

Fig. 8.
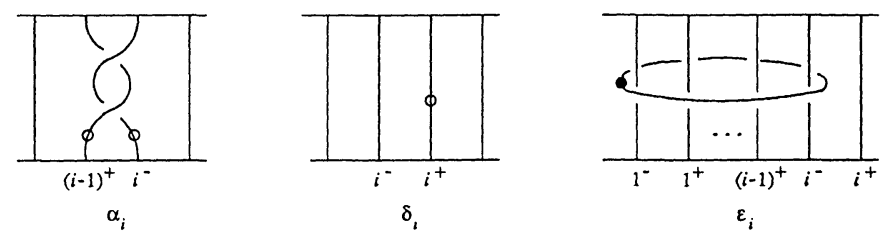

Remark 4.1. Applying the move $K_{3}^{-1}$, the regular isotopy, and the move $K_{3}$ successively, we can shift the white circle (the positive kink) of $\delta_{2}$ from the string $i^{+} \times[0,1]$ to the string $i^{-} \times[0,1]$.

Theorem 4.1 (Main Theorem). There exists an isomorphism $\phi: T_{2 n} \rightarrow M_{n, 1}$ which maps $\alpha_{i}, \beta_{i}, \delta_{i}, \varepsilon_{i}$ to $a_{\imath}, b_{\imath}, d_{i}, e_{i}, 1 \leq i \leq n$ respectively.

Remark 4.2. One may obtain a similar result for the mapping class group $M_{n, 0}$ of a closed surface, taking the factor of $T_{2 n}$ by cyclic commutator subgroup generated by full twist on $2 n$ strings.

Remark 4.3. This tangle presentation of $M_{n, 1}$ has a straightforward generalization to the case of mapping class group $M_{n, 1, k}$ of a surface with $k$ marked points by introducing $k$ new strands with braiding around the other strings and between themselves (but without any new $\beta_{i}$ generators). 


\section{Proof of the Main Theorem: Construction of $\phi$}

Consider a large disk $B \subset \mathbb{R}^{2}$ containing $Y_{2 n}$. Without loss of generality we may assume that all considered $2 n$-tangles are contained in $B \times[0,1]$. Let $\xi \subset B \times[0,1]$ be an admissible $2 n$-tangle. For each pair $\left(i^{-}, i^{+}\right) \subset Y_{2 n}$ attach to $B \times[0,1]$ index one handle $N\left(A_{\imath}\right) \subset \mathbb{R}^{2} \times \mathbb{R}^{1}=\mathbb{R}^{3}$ so that $N\left(A_{i}\right) \cap B \times[0,1]$ is the regular neighbourhood of $\left(i^{-} \cup i^{+}\right) \times\{1\}$. Add to $\xi$ the cores $A_{i}$ of the handles to close $\xi$ from above. To make the construction explicit we will take $A_{i}=\left\{(0, y, z) \in \mathbb{R}^{3} \mid(y-i)^{2}+(z-1)^{2}=\right.$ $1 / 4, z \geq 1\}$. We obtain the solid handlebody $H=B \times[0,1] \cup\left(\bigcup_{i} N\left(A_{i}\right)\right) \subset \mathbb{R}^{3}$ with framed one-dimensional submanifold $\tilde{\xi}=\xi \cup\left(\bigcup_{i} A_{i}\right)$ inside it. Denote by $\xi^{\prime}$ the union of all unclosed components of $\tilde{\xi}$. It consists of exactly $n$ arcs with the ends on the "bottom" $B \times\{0\}$ of $H$. Finally, remove from $H$ interior of a regular neighbourhood $N\left(\xi^{\prime}\right)$ of $\xi^{\prime}$. As a result we obtain 3-manifold $H_{\xi}=H \backslash \operatorname{Int}\left(N\left(\xi^{\prime}\right)\right)$ with framed link $L_{\xi}=\tilde{\xi} \backslash \xi^{\prime}$ inside it, see Fig. 9. Note that $\partial H_{\xi}$ admits natural decomposition $\partial H_{\xi}=\Sigma_{0 \xi} \cup \partial B \times[0,1] \cup \Sigma_{1 \xi}$, where $\Sigma_{\jmath \xi}, j=0,1$ is genus $n$ surface with one boundary component and $\Sigma_{j \xi} \cap \partial B \times[0,1]=\partial B \times\{j\}$.

We would like to point out that there exist natural identifications $\kappa_{j}: \Sigma_{n, 1} \rightarrow \Sigma_{\jmath \xi}$. Present $\Sigma_{n, 1}$ as disk $B$ with $n$ handles as it is depicted in Fig. 10 (compare with Fig. 7). The surface $\Sigma_{j \xi}$ is also disk $B \times\{j\}$ with $n$ handles. Then $\kappa_{\jmath}$ maps $B$ with holes identically to $B \times\{j\}$ with holes and the curves $d_{i}, b_{i}$ are mapped, respectively, to the meridians and induced by framing "longitudes" of the corresponding handles (the images of $b_{\imath}$ in Fig. 9 are drawn by thick lines).

Recall that $\chi\left(H_{\xi}, L_{\xi}\right)$ denotes 3-manifold obtained from $H_{\xi}$ by surgery on the framed link $L_{\xi} \subset H_{\xi}$.

Proposition 5.1. For any admissible $2 n$-tangle $\xi$ the manifold $\chi\left(H_{\xi}, L_{\xi}\right)$ is homeomorphic to $\Sigma_{n, 1} \times[0,1]$ and the product structure induced on $\chi\left(H_{\xi}, L_{\xi}\right)$ is an extension of the natural product structure on $\partial B \times[0,1] \subset \partial H_{\xi}=\partial \chi\left(H_{\xi}, L_{\xi}\right)$.

Proof. Since the complement of a braid in $B \times[0,1]$ is homeomorphic to the complement of the trivial braid, the proposition holds for the case when $\xi$ is an

Fig. 9.
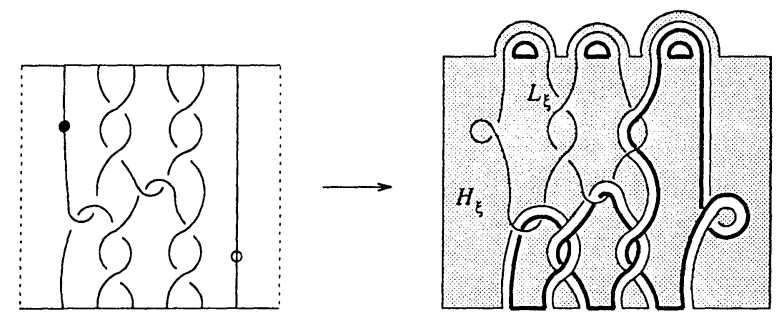

$\xi$

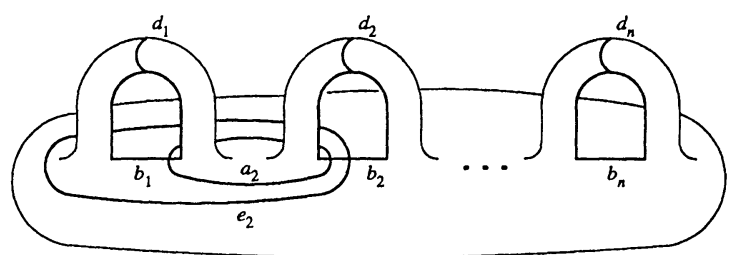

Fig. 10 . 
Fig. 11.
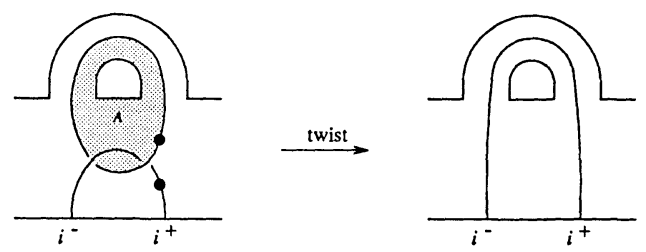

admissible $2 n$-braid. Using the twist along the annulus $A \subset H_{\xi}$, one boundary component of which coincides with $\kappa_{1}\left(b_{\imath}\right) \subset \Sigma_{1 \xi} \subset \partial H_{\xi}$ and another - with the unique closed component of $L_{\beta_{i}}^{ \pm 1}$, one can easily prove the proposition for $\xi=\beta_{i}^{ \pm 1}$, as shown in Fig. 11. It remains to note that by the definition of admissible $2 n$-tangle product structure on $\chi\left(H_{\xi \zeta}, L_{\xi \zeta}\right)$ is obtained by gluing together the product structures on $\chi\left(H_{\xi}, L_{\xi}\right)$ and $\chi\left(H_{\zeta}, L_{\zeta}\right)$, which implies the general case.

Consider an admissible $2 n$-tangle $\xi$. Recall that $\partial \chi\left(H_{\xi}, L_{\xi}\right)=\partial H_{\xi}=\Sigma_{0 \xi} U$ $\partial B \times[0,1] \cup \Sigma_{1 \xi}$. Let $p_{\xi}: \Sigma_{0 \xi} \rightarrow \Sigma_{1 \xi}$ be the restriction on $\Sigma_{0 \xi}$ of the direct product projection $p: \partial \chi\left(H_{\xi}, L_{\xi}\right) \rightarrow \Sigma_{1 \xi}$. Define the homeomorphism $\phi(\xi): \Sigma_{n, 1} \rightarrow \Sigma_{n, 1}$ by $\phi(\xi)=\kappa_{1}^{-1} p_{\xi} \kappa_{0}$. It follows from the definition that $\phi(\xi \zeta)=\phi(\xi) \phi(\zeta)$ for any two admissible $2 n$-tangles $\xi, \zeta$.

Remark 5.1. The homeomorphisms $\phi\left(\beta_{i}^{ \pm 1}\right)$ are isotopic to the twists along the curve $b_{\imath}$ in positive and negative directions respectively, see Fig. 10 and the proof of Proposition 5.1.

Proposition 5.2. The assignment $\xi \mapsto \phi(\xi)$ determines correctly defined homomorphism $\phi: T_{2 n} \rightarrow M_{n, 1}$.

Proof. It is sufficient to show that the isotopy class of $\phi(\xi)$ depends only on the $K$ equivalence class of $\xi$. Let an admissible $2 n$-tangle $\zeta$ be obtained from $\xi$ by application of the move $K=K_{1}, K_{2}$ or $K_{3}$. Suppose that all the components of $\xi$ involved in the move $K$ are closed. Then the equality $\phi(\xi)=\phi(\zeta)$ is clear: the same proof as for the easy "only if" part of the Kirby theorem is valid. But all components of $\xi$ are actually closed in $2 n$-tangle $\Delta \xi \Delta$, where $\Delta=\prod_{i=1}^{n} \beta_{i} \beta_{\imath}^{-1}$. Therefore $\phi(\Delta \xi \Delta)=\phi(\Delta \zeta \Delta)$. The multiplicativity of $\phi$ and the equality $\phi(\Delta)=1$ (see Remark 5.1) imply the desired $\phi(\xi)=\phi(\zeta)$ thus completing the proof.

By the construction of $\phi$ we obtain that $\phi$ maps the tangles $\alpha_{i}, \beta_{\imath}, \delta_{\imath}, \varepsilon_{\imath}$ to $a_{\imath}, b_{\imath}$, $d_{\imath}, e_{\imath}, 1 \leq i \leq n$ respectively.

\section{Proof of the Main Theorem: Construction of $\psi=\phi^{-1}$}

Let us begin with reformulation of Wajnryb's theorem [13].

Theorem [13]. The mapping class group $M_{n, 1}$ admits a presentation with generators $a_{1}, b_{1}, \ldots, a_{n}, b_{n}, e_{2}$ and relations

(A) $a_{\imath} b_{i} a_{\imath}=b_{i} a_{\imath} b_{\imath}, a_{\imath+1} b_{\imath} a_{i+1}=b_{i} a_{\imath+1} b_{\imath}, b_{2} e_{2} b_{2}=e_{2} b_{2} e_{2}$, every other pair of generators commute;

(B) $\left(a_{2} b_{1} a_{1}\right)^{4}=k e_{2} k^{-1} e_{2}$, where $k=b_{2} a_{2} b_{1} a_{1}^{2} b_{1} a_{2} b_{2}$;

(C) $a_{1}^{-1} a_{2}^{-1} a_{3}^{-1} g_{1} g_{2} e_{2}=w e_{2} w^{-1}$, where $t_{1}=b_{1} a_{2} a_{1} b_{1}, t_{2}=b_{2} a_{3} a_{2} b_{2}, g_{2}=t_{2}^{-1} e_{2} t_{2}$, $g_{1}=t_{1}^{-1} g_{2} t_{1}, u=a_{3}^{-1} b_{3}^{-1} g_{2} b_{3} a_{3}, w=b_{3} a_{3} b_{2} a_{2} b_{1} u a_{1}^{-1} b_{1}^{-1} a_{2}^{-1} b_{2}^{-1}$. 
Remark 6.1. Our formulation of Wajnryb's theorem differs slightly from the original one: we write homeomorphisms from right to left and use $\varepsilon_{2}$ instead of $\delta_{2}$. The latter is possible due to existence of rotation of $\Sigma_{n, 1}$ which is invariant on $\alpha_{i}, \beta_{i}$ and interchanges $\delta_{2}$ and $\varepsilon_{2}$.

Proposition 6.1. The assignment $a_{i} \mapsto \alpha_{i}, b_{i} \mapsto \beta_{i}, e_{i} \mapsto \varepsilon_{i}$ determines correctly defined homomorphism $\psi: M_{n, 1} \rightarrow T_{2 n}$.

Proof. We need only check that this assignment transforms the relations (A), (B), (C) to true equalities on the tangle level.

(A) The equality $\alpha_{i} \beta_{i} \alpha_{i}=\beta_{i} \alpha_{i} \beta_{i}$ is verified in Fig. 12 . Verification of the equality $\alpha_{i+1} \beta_{\imath} \alpha_{\imath+1}=\beta_{i} \alpha_{i+1} \beta_{i}$ is similar. Equality $\beta_{2}^{-1} \varepsilon_{2} \beta_{2}=\varepsilon_{2} \beta_{2} \varepsilon_{2}^{-1}$ (equivalent to $\beta_{2} \varepsilon_{2} \beta_{2}=\varepsilon_{2} \beta_{2} \varepsilon_{2}$ ) is verified in Fig. 13. Obviously every other pair of $2 n$-tangles $\alpha_{i}, \beta_{2}, \varepsilon_{2}$ commutes.

(B) Let $\kappa=\beta_{2} \alpha_{2} \beta_{1} \alpha_{1}^{2} \beta_{1} \alpha_{2} \beta_{2}$ be the image of $k$.

From Fig. 14 we obtain that $\kappa \varepsilon_{2}=\delta_{2} \kappa$. Therefore $\kappa \varepsilon_{2} \kappa^{-1}=\delta_{2}$. Using (A) we deduce

$$
\left(\alpha_{2} \beta_{1} \alpha_{1}\right)^{4}=\left(\alpha_{2} \beta_{1} \alpha_{1} \alpha_{2} \beta_{1} \alpha_{1}\right)^{2}=\left(\beta_{1} \alpha_{2} \beta_{1} \alpha_{1} \beta_{1} \alpha_{1}\right)^{2}=\left(\beta_{1} \alpha_{2} \alpha_{1} \beta_{1} \alpha_{1}^{2}\right)^{2} .
$$

Fig. 12. $\beta_{i} \alpha_{i} \beta_{i}=$
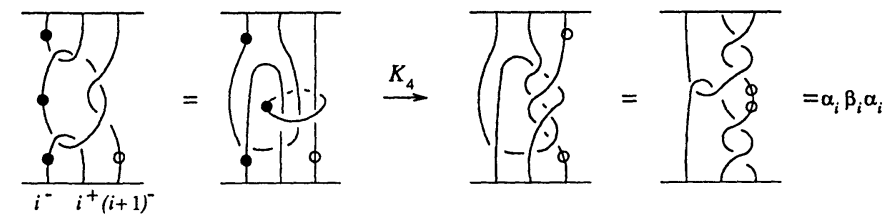

Fig. 13.

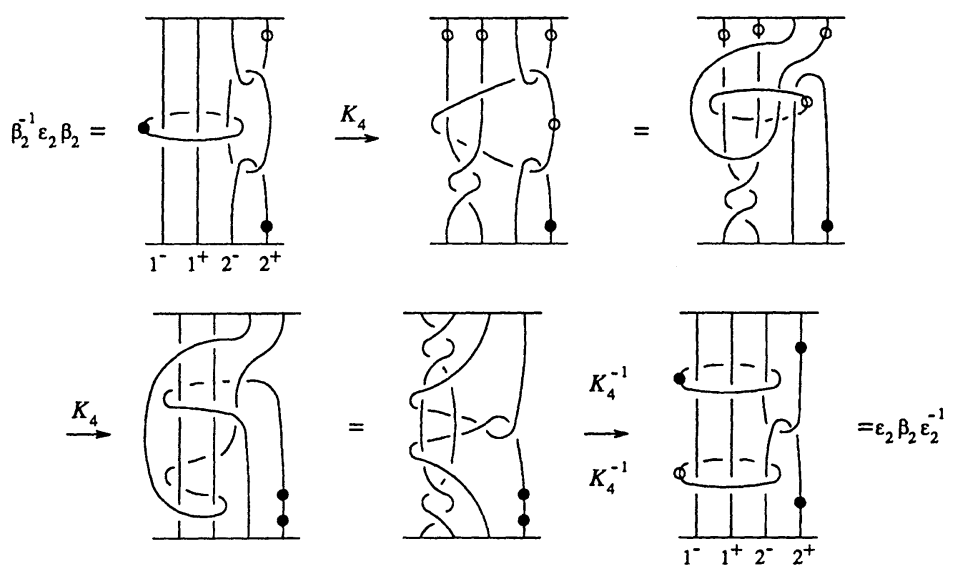

Fig. 14.
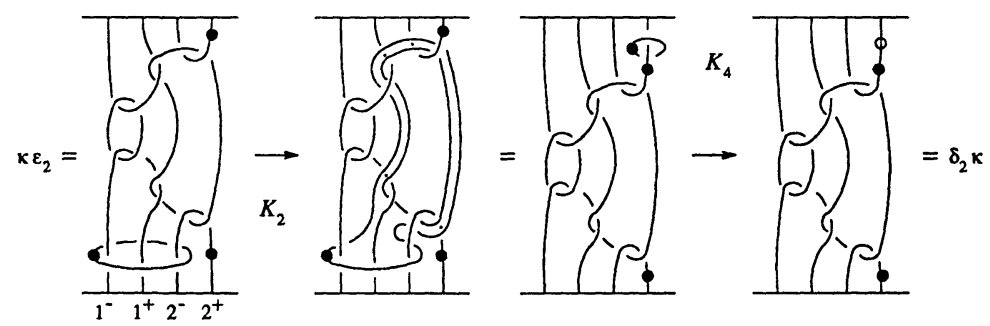
Fig. 15.

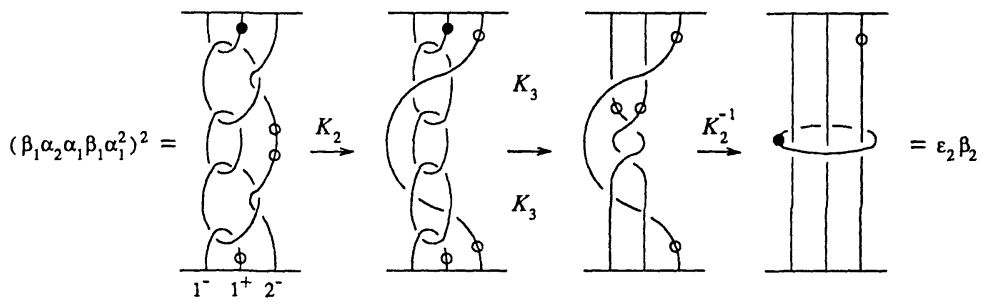

Fig. 16.

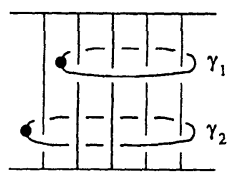

Fig. 17.
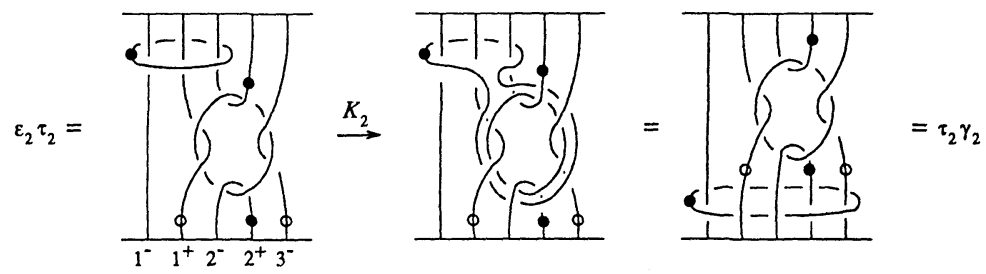

Fig. 18.
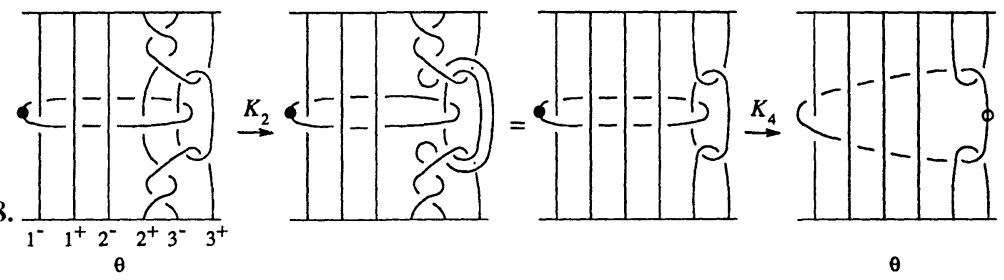

Fig. 19.
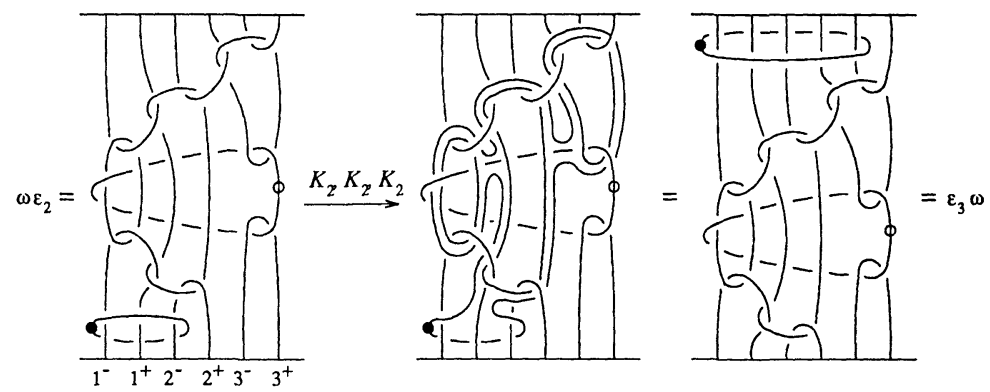

From Fig. 15 we now obtain $\left(\beta_{1} \alpha_{2} \alpha_{1} \beta_{1} \alpha_{1}^{2}\right)^{2}=\delta_{2} \varepsilon_{2}$ which implis (B).

(C) Let $\gamma_{1}, \gamma_{2}$ be the tangles depicted in Fig. 16. It follows from Fig. 17 that $\tau_{2} \gamma_{2}=\varepsilon_{2} \tau_{2}$, where $\tau_{2}=\beta_{2} \alpha_{3} \alpha_{2} \beta_{2}$ is the image of $t_{2}$. Hence we have $\gamma_{2}=\tau_{2}^{-1} \varepsilon_{2} \tau_{2}$. A similar trick enables us to prove that $\gamma_{1}=\tau_{1}^{-1} \gamma_{2} \tau_{1}$ for the image $\tau_{1}=\beta_{1} \alpha_{2} \alpha_{1} \beta_{1}$ of $t_{1}$. We may conclude, therefore, that the tangles $\gamma_{1}, \gamma_{2}$ serve as the images of $g_{1}, g_{2}$ respectively.

To express the tangle $\theta=\alpha_{3}^{-1} \beta_{3}^{-1} \gamma_{2} \beta_{3} \alpha_{3}$ (the image of $u$ ) in a more convenient form we apply the moves $K_{2}$ and $K_{4}$ as shown in Fig. 18. We deduce from Fig. 19 that 
Fig. 20.

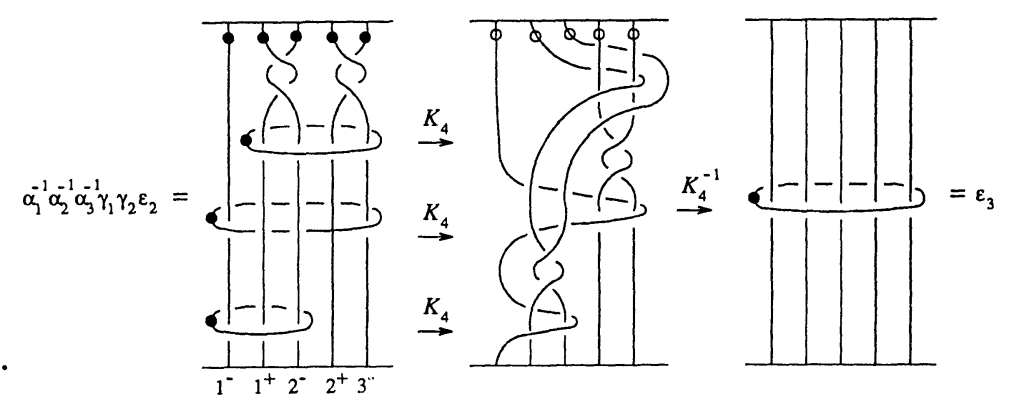

the image $\omega \varepsilon_{2} \omega^{-1}$ (where $\omega=\beta_{3} \alpha_{3} \beta_{2} \alpha_{2} \beta_{1} \theta \alpha_{1}^{-1} \beta_{1}^{-1} \alpha_{2}^{-1} \beta_{2}^{-1}$ ) of the RHS of relation (C) equals $\varepsilon_{3}$. The image of the LHS of relation (C) also equals $\varepsilon_{3}$, as shown in Fig. 20.

This completes the proof of the proposition.

The Main Theorem now follows from Propositions 5.2, 6.1.

\section{From Heegard Diagrams to Surgery}

Let $H_{n}$ denote the standard handlebody of genus $n$ in $\mathbb{R}^{2} \times \mathbb{R}^{1} \subset S^{3}$. We shall consider a Heegard diagram of 3-manifold $M$ as a homeomorphism $h: \partial H_{n} \rightarrow \partial H_{n}$ such that $\overline{\left(S^{3} \backslash H_{n}\right)} \cup_{h} H_{n}=M$. Identify $\Sigma_{n, 1}$ with the complement of an open disk $D \subset \partial H_{n}$ and the group $M_{n, 1}$ with the mapping class group of $\partial H_{n}$ modulo $D$. Particularly, one may assume that $h \subset M_{n, 1}$. Let $\xi=\psi(h)$ be the admissible $2 n$-tangle corresponding to $h$. One may express $\xi$ as a product of tangles $\alpha_{i}, \beta_{i}, \delta_{\imath}$ according to a decomposition of $h$ into the product of twists along the curves $a_{i}, b_{i}, d_{i}$.

Recall that the framed link $L_{\xi} \subset H_{\xi} \subset S^{3}$ is obtained from $\xi$ by closing with $n$ small semicircles from above and removing the lower strings.

Theorem 7.1. For any admissible $2 n$-tangle $\xi$ the manifolds $\chi\left(S^{3}, L_{\xi}\right)$ and

$$
\left(S^{3} \backslash H_{n}\right) \cup_{\phi(\xi)} H_{n}
$$

are homeomorphic.

Proof. Let $B_{1} \subset \mathbb{R}^{2}$ be a disk such that Int $B_{1} \subset B$. Denote by $H_{\xi}^{0}$ the handlebody $B_{1} \times[-1 / 2,0] \cup N\left(\xi^{\prime}\right) \subset \mathbb{R}^{2} \times \mathbb{R}^{1} \subset S^{3}$ and by $H_{\xi}^{1}$ the handlebody $B \times$ $[-1,1] \cup\left(\bigcup_{i=1}^{n} N\left(A_{2}\right)\right)$, see Fig. 21 and Sect. 5. It follows from Proposition 5.1 that $\chi\left(\overline{\left(H_{\xi}^{1} \backslash H_{\xi}^{0}\right)}, L_{\xi}\right)$ is homeomorphic to $\partial H_{\xi}^{0} \times[0,1]$. Let $p_{\xi}: \partial H_{\xi}^{0} \rightarrow \partial H_{\xi}^{1}$

Fig. 21.

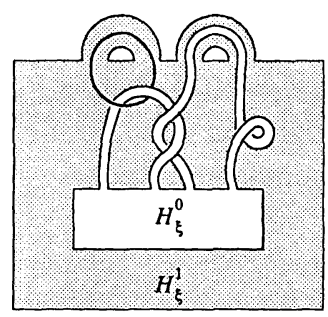


be the restriction of the direct product projection. Clearly the manifold $\chi\left(S^{3}, L_{\xi}\right)$ is homeomorphic to $\overline{\left(S^{3} \backslash H_{\xi}^{1}\right)} \cup p_{\xi} H_{\xi}^{0}$. Homeomorphisms $\kappa_{0}: \Sigma_{n, 1} \rightarrow \Sigma_{0 \xi}$ and $\kappa_{1}: \Sigma_{n, 1} \rightarrow \Sigma_{1 \xi}$ defined in Sect. 5 can be extended to homeomorphisms $\tilde{\kappa}_{0}: H_{n} \rightarrow H_{\xi}^{0}$ and $\tilde{\kappa}_{1}: \overline{\left(S^{3} \backslash H_{n}\right)} \rightarrow \overline{\left(S^{3} \backslash H_{\xi}^{1}\right)}$ respectively. Since $\kappa_{1}^{-1} p_{\xi} \kappa_{0}=\phi(\xi)$ by definition of $\phi$, the formulas $f(x)=\tilde{\kappa}_{0}(x)$ if $x \in H_{n}$ and $f(x)=\tilde{\kappa}_{1}(x)$ if $x \in \overline{\left(S^{3} \backslash H_{n}\right)}$ give correctly defined homeomorphism $f: \overline{\left(S^{3} \backslash H_{n}\right)} \cup_{p_{\xi}} H_{n} \rightarrow \overline{\left(S^{3} \backslash H_{\xi}^{1}\right)} \cup_{p_{\xi}} H_{\xi}^{0} \approx \chi\left(S^{3}, L_{\xi}\right)$.

We now briefly recall the notion of arborescent link (see [3]). Let $\Gamma \subset \mathbb{R}^{2}$ be connected multigraph. Suppose that there exist a disk $D \subset \mathbb{R}^{2}$ such that $D \cap \Gamma=\partial D$ and $\partial D$ consists of exactly two vertices and two edges of $\Gamma$. Collapsing $D$ to a point we obtain a new multigraph $\Gamma^{\prime} \subset \mathbb{R}^{2}$ as shown in Fig. 22.

Fig. 22.

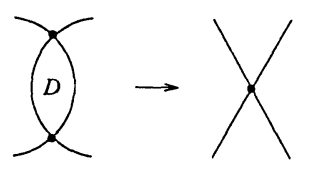

Definition. A link $L \subset \mathbb{R}^{3}$ is called arborescent, if it admits a (general position) projection $\tilde{L} \subset \mathbb{R}^{2}$ which, if considered as multigraph, can be reduced to the onevertex multigraph (i.e. to figure eight) by a sequence of transformations described above.

Corollary. Any 3-manifold of Heegard genus two may be obtained from $S^{3}$ by surgery on a framed arborescent link.

Proof. Let $\xi$ be an admissible 4-tangle presented as a product of $\alpha_{1}, \alpha_{2}, \beta_{1}, \beta_{2}, \delta_{2}$ and let $L_{\xi}$ be the corresponding link. One may easily deduce that $L_{\xi}$ is arborescent (its natural projection becomes arborescent multigraph after untwisting small kinks). The corollary now follows from the Main Theorem and Theorem 7.1.

\section{From Surgery to Heegard Diagrams}

Theorem 8.1. For each framed link $L \subset S^{3}$ there exist $n$ and an admissible $2 n$-tangle $\xi$, such that $L$ may be transformed into $L_{\xi}$ by a sequence of Kirby moves. Tangle $\xi$ may be chosen in the form $\xi=\eta \pi_{n}$, where $\pi_{n}=\prod_{i=1}^{n} \delta_{i} \beta_{i} \delta_{i}$ and $\eta$ is a pure $2 n$-braid.

Remark 8.1. The first part of the theorem is an easy consequence of the Kirby theorem, the Main Theorem and Theorem 7.1, but we will provide a constructive proof without using the Kirby theorem.

Remark 8.2. Theorem 8.1 allows one to construct a Heegard diagram of 3-manifold $M$ starting from any surgery presentation: from $M=\chi\left(S^{3}, L\right)$ we pass to $M=\chi\left(S^{3}, L_{\xi}\right)$ and then to the presentation $M=\overline{\left(S^{3} \backslash H_{n}\right)} \cup_{p_{\xi}} H_{n}$ by Theorem 7.1.

Definition $[1,10]$. An $n$-component link $L \subset \mathbb{R}^{3}$ is said to be represented by pure $2 n$-plat if it admits a diagram with $n$ local maxima (with respect to the projection on the $z$-axis). 
Remark 8.3. Each pure $2 n$-plat is regularly isotopic to the plat closure of pure $2 n$ braid.

Let us prove the following

Lemma 8.1. Each framed link can be transformed to a pure $2 n$-plat by a sequence of Kirby moves.

Proof. Denote by $m(L)$ the number of local maxima and by $n(L)$ the number of components of $L$. Suppose that $d(L)=m(L)-n(L)>0$. Then there exists a component $L_{\imath}$ of $L$ with at least two points of local maxima. Using the move $K_{3}^{-1}$ we may pass to a link $L^{\prime}$ with $d\left(L^{\prime}\right)=d(L)-1$ as shown in Fig. 23 (the diagrams coincide inside the square). After $d(L)$ steps we will obtain a pure $2 n$-plat.

Fig. 23.

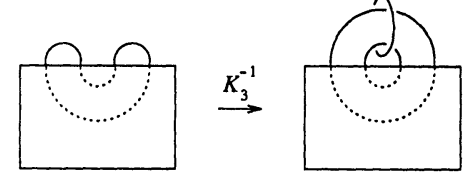

We are in a position to prove Theorem 8.1.

Proof of Theorem 8.1. By Lemma 8.1 transform $L$ to a pure $2 n$-plat $L^{\prime}$ which is a plat closure of some pure $2 n$-braid $\eta$. Then $\xi=\eta \pi_{n}$ satisfies the theorem since $L_{\xi}=L^{\prime}$ by the definition of $L_{\xi}$ and the proof is complete.

The following corollary can be considered as a version of Birman's theorem on the existence of special Heegard diagrams $[2,10]$ :

Corollary. Each closed orientable 3-manifold can be obtained by pasting together two copies of the standard handlebody $H_{n} \subset S^{3}$ via a homeomorphism $u: \partial H_{n} \rightarrow \partial H_{n}$ which is fixed on all longitudes $b_{i}, 1 \leq i \leq n$ (and therefore is extendable to $\overline{\left(S^{3} \backslash H_{n}\right)}$ ).

Proof. From Remark 8.2 we obtain that $M=\overline{\left(S^{3} \backslash H_{n}\right)} \cup_{h} H_{n}$, where homeomorphism $h: \partial H_{n} \rightarrow \partial H_{n}$ has the form $h=\phi\left(\eta \pi_{n}\right)$ for some pure $2 n$-braid $\eta$. One can easily verify that the homeomorphism $p_{n}=\phi\left(\pi_{n}\right)$ maps each meridian $d_{i} ; 1 \leq i \leq n$ to the corresponding longitude $b_{i}$ and vice versa and, hence, may be extended to homeomorphism of $\overline{\left(S^{3} \backslash H_{n}\right)}$ onto $H_{n}$. This implies that $M$ is homeomorphic to $H_{n} \cup_{u} H_{n}$ for $u=p_{n}^{-1} h$. Since $\eta$ is a pure braid, homeomorphism $\phi(\eta)$ preserves meridians. Therefore homeomorphism $u=p_{n}^{-1} h=p_{n}^{-1} \phi(\eta) p_{n}$ preserves longitudes and the corollary follows.

\section{Proof of the Kirby Theorem}

We start with some preliminary lemmas.

Lemma 9.1. Let $\xi, \zeta$ be $K$-equivalent admissible $2 n$-tangles. Then the corresponding links $L_{\xi}, L_{\zeta}$ are $K$-equivalent.

Proof. Let $\zeta$ be obtained from $\xi$ by the move $K=K_{1}, K_{2}$ or $K_{3}$. Assume first that components of $\xi$ with the ends on the bottom $B \times\{0\}$ of $B \times[0,1]$ do not take part in $K$. Then $L_{\zeta}$ is obtained from $L_{\xi}$ by the same move $K$. If $K=K_{2}$ or $K_{3}$ and components of $\xi$ with the ends on $B \times\{0\}$ do take part in $K$, then $L_{\zeta}$ either coincides with $L_{\xi}$ or is obtained from $L_{\xi}$ by the move $K_{5}$. 
The following two lemmas assure us that multiplication of $\xi$ by a tangle corresponding to a homeomorphism extendable to the inner or outer handlebody does not change $K$-equivalence class of $L_{\xi}$.

Denote by $T I_{2 n} \subset T_{2 n}$ a subgroup of admissible $2 n$-tangles corresponding (via $\phi)$ to homeomorphisms of $\partial H_{n}$ which are extendable to $H_{n}$.

Lemma 9.2. Let $\gamma_{I}$ be an arbitrary tangle in the subgroup $T I_{2 n}$. Then for any admissible $2 n$-tangle $\xi$ links $L_{\xi}$ and $L_{\zeta}$ for $\zeta=\xi \gamma_{I}$ are $K$-equivalent.

Proof. Obviously, all admissible $2 n$-braids belong to $T I_{2 n}$ since the corresponding homeomorphisms of $\partial H_{n}$ are invariant on the union $\bigcup_{i=1}^{n} d_{i}$ of meridians of $H_{n}$ (and vice versa, any homeomorphism which is invariant on the union of meridians may be obtained as $\phi(\eta)$ for some admissible $2 n$-braid $\eta)$. Moreover, one can easily check that $2 n$-tangle $\tau_{1}=\beta_{1} \alpha_{2} \alpha_{1} \beta_{1}$ also belongs to $T I_{2 n}$ since the corresponding homeomorphism $t_{1}=\phi\left(\tau_{1}\right): \partial H_{n} \rightarrow \partial H_{n}$ maps $d_{1}$ to $a_{2}$ and is fixed on all other meridians $d_{2}, d_{3}, \ldots, d_{n}$. It is known that each homeomorphism of $\partial H_{n}$ which is extendable to $H_{n}$ can be expressed via $t_{1}$ and homeomorphisms of $\partial H_{n}$ which are invariant on the union $\bigcup_{i=1}^{n} d_{i}$ of meridians of $H_{n}$ (see $[12,8]$ ). Therefore the subgroup $T I_{2 n}$ is generated by admissible $2 n$-braids and $\tau_{1}$ and it suffices to check the statement of the lemma for $\gamma_{I}$ being admissible $2 n$-braid and for $\gamma_{I}=\tau_{1}$. If $\gamma_{I}$ is an admissible $2 n$-braid then $L_{\zeta}=L_{\xi}$ by the construction of $L_{\zeta}$ (removing of the lower strings of $\zeta$ removes all strings of $\gamma_{I}$ ). For $\gamma_{I}=\tau_{1}$ we may obtain $L_{\xi}$ from $L_{\zeta}$ by the move $K_{5}$ and the lemma follows.

Denote by $\mathrm{TO}_{2 n} \subset T_{2 n}$ a subgroup of admissible $2 n$-tangles corresponding (via $\phi)$ to homeomorphisms of $\partial H_{n}$ which are extendable to $\overline{\left(S^{3} \backslash H_{n}\right)}$.

Lemma 9.3. Let $\gamma_{O}$ be an arbitrary tangle in the subgroup $T O_{2 n}$. Then for any admissible $2 n$-tangle $\xi$ links $L_{\xi}$ and $L_{\zeta}$ for $\zeta=\gamma_{O} \xi$ are $K$-equivalent.

Proof. Recall that homeomorphism $p_{n}=\phi\left(\pi_{n}\right)$ where $\pi_{n}=\prod_{i=1}^{n} \delta_{\imath} \beta_{i} \delta_{i}$ permutes each meridian $d_{i}$ with the corresponding longitude $b_{i}$ and, hence, may be extended to homeomorphisms of $H_{n}$ to $\overline{\left(S^{3} \backslash H_{n}\right)}$ and of $\overline{\left(S^{3} \backslash H_{n}\right)}$ to $H_{n}$. This implies that $\pi_{n} \gamma_{O} \pi_{n}^{-1}$ belongs to $T I_{2 n}$ (which is generated by admissible $2 n$-braids and $\tau_{1}$ ). So it is sufficient to prove the lemma for $\gamma_{O}=\pi_{n}^{-1} \gamma_{I} \pi_{n}$, where $\gamma_{I}$ is either admissible $2 n$-braid or $\tau_{1}$. In the first case the link $L_{\xi}$ is obtained from $L_{\zeta}$ by $n$ moves $K_{5}$, in the second - by $n+1$ moves $K_{5}$ as shown in Fig. 24 .

Fig. 24 .
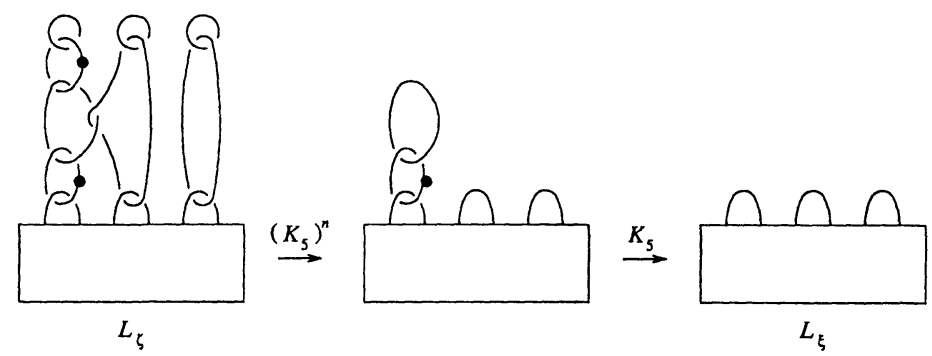
Note that for any $m, n$ there exists a natural embedding $i_{m, n}: T_{2 m} \rightarrow T_{2 n}$ generated by the addition of $2(n-m)$ new vertical strings (of the form $\left\{i^{ \pm}\right\} \times[0,1] \subset \mathbb{R}^{2} \times[0,1]$, $m+1 \leq i \leq n$ ) to each admissible $2 n$-tangle $\xi$. The addition of new strings does not change $L_{\xi}$. This observation motivates the following

Definition. Two admissible tangles $\xi \in T_{2 m}, \zeta \in T_{2 n}$ are called stably equivalent (we write $\xi \underset{\text { st }}{\tilde{\zeta}}$ ), if $i_{m, N}(\xi)=\gamma_{O} i_{n, N}(\zeta) \gamma_{I}$ for some $N \geq m$, n and $\gamma_{O} \in T O_{2 N}$, $\gamma_{I} \in T I_{2 N}$.

Lemma 9.4. If $\xi \underset{s t}{\sim} \zeta$, then $L_{\xi} \underset{K}{\sim} L_{\zeta}$.

Proof. Immediately follows from Lemmas 9.1-9.3.

Proof of the Kirby theorem. Let $L_{1}, L_{2} \subset S^{3}$ be two links such that $\chi\left(S^{3}, L_{1}\right)=$ $\chi\left(S^{3}, L_{2}\right)$. Using Theorem 8.1 we may construct (for some $m, n$ ) admissible tangles $\xi \in T_{2 m}, \zeta \in T_{2 n}$ so that $L_{1} \underset{K}{\sim} L_{\xi}, L_{2} \underset{K}{\sim} L_{\zeta}$. Obviously we have $\chi\left(S^{3}, L_{\xi}\right)=\chi\left(S^{3}, L_{\zeta}\right)$ which (by Theorem 7.1) is equivalent to $\overline{\left(S^{3} \backslash H_{m}\right)} \cup_{\phi(\xi)} H_{m}=\overline{\left(S^{3} \backslash H_{n}\right)} \cup_{\phi(\zeta)} H_{n}$. Now use the Reidemeister-Singer theorem [9] which states that any two Heegard diagrams of the same 3-manifold are stably equivalent. Translating this theorem to admissible tangle setting by means of Theorem 4.1 we obtain that tangles $\xi$ and $\zeta$ are stably equivalent. It follows from Lemma 9.4 that $L_{\xi} \underset{K}{\sim} L_{\zeta}$ which implies the theorem.

\section{References}

1. Birman, J.: Braids, links and mapping class groups. Ann. Math. Studies 82, 3-227 (1975)

2. Birman, J.: Special Heegard splittings for closed oriented 3-manifolds. Topology 17, 157-166 (1978)

3. Caudron, A.: Classification des noeuds et des enlacements. Notes de recherche 76-80, Publ. Math. D’Orsay 82-04, 350p.

4. Fenn, R., Rourke, C.: On Kirby's calculus of links. Topology 18, 1-15 (1979)

5. Kirby, R.C.: A calculus for framed links in $S^{3}$. Invent. Math. 45, 35-56 (1978)

6. Lickorish, W.B.R.: A representation of orientable combinatorial 3-manifolds. Ann. Math. 76, 531-540 (1962)

7. Ning Lu: A simple proof of the fundamental theorem of Kirby calculus on links. Trans. Am. Math. Soc. 331, 143-156 (1992)

8. Matveev, S., Fomenko, A.: Algorithmical and computer methods in 3-manifold topology. Moscow: Moscow University Press 1991, 300p. (Russian)

9. Reidemeister, K.: Zur dreidimensionalen Topologie. Ann. Math. Sem. Univ. Hamburg 9, 184-194 (1983)

10. Rego, E., de Sa, E.C.: Special Heegard diagrams and the Kirby calculus. Topology and its Appl. 37, 11-24 (1990)

11. Reshetikhin, N.Yu., Turaev, V.G.: Ribbon graphs and their invariants derived from quantum groups. Commun. Math. Phys. 127, 1-26 (1990)

12. Suzuki, S.: On homeomorphisms of a 3-dimensional handlebodies. Can. J. Math. 29, no. 4, 111-129 (1977)

13. Wajnryb, B.: A simple presentation for the mapping class group of an orientable surface. Israel J. Math. 45, 157-174 (1983) 